\title{
Laquinimod in the treatment of multiple sclerosis: a review of the data so far
}

\author{
This article was published in the following Dove Press journal: \\ Drug Design, Development and Therapy \\ 14 March 2016 \\ Number of times this article has been viewed
}

\author{
Jan Thöne' \\ Ralf A Linker ${ }^{2}$ \\ 'Department of Neurology, University \\ Hospital Essen, Essen, ${ }^{2}$ Department \\ of Neurology, University Hospital \\ of Erlangen, Friedrich-Alexander \\ University Erlangen-Nürnberg (FAU), \\ Erlangen, Germany
}

\begin{abstract}
Laquinimod (ABR-215062) is a new orally available carboxamide derivative, which is currently developed for relapsing remitting (RR) and chronic progressive (CP) forms of multiple sclerosis (MS; RRMS or CPMS) as well as neurodegenerative diseases. Its mechanism of action may comprise immunomodulatory effects on T-cells, monocytes, and dendritic cells as well as neuroprotective effects with prominent actions on astrocytes. Laquinimod was tested in Phase II and III clinical trials in RRMS at different dosages ranging from 0.1 to $0.6 \mathrm{mg} /$ day. The compound was well tolerated, yet at the dosages tested only led to moderate effects on the reduction of relapse rates as primary study endpoint in Phase III trials. In contrast, significant effects on brain atrophy and disease progression were observed. While there were no significant safety signals in the clinical trials, the Committee for Medicinal Products for Human Use (CHMP) refused marketing authorization for RRMS based on the assessment of the risk-benefit ratio with regard to data from animal studies. At present, the compound is further tested in RRMS as well as CPMS and Huntington's disease at different concentrations. Results from these trials will further inform about the clinical benefit of laquinimod in patient cohorts with a persisting, but still insufficiently met need for safe and at the same time effective oral compounds with neuroprotective effects.
\end{abstract}

Keywords: neuroprotection, ABR-215062, axonal damage, demyelination

\section{Introduction}

Multiple sclerosis (MS) is a chronic and degenerative immune mediated disorder of the central nervous system (CNS) often affecting young adults and requiring long-term treatment. ${ }^{1,2}$ The disease course varies considerably between individuals and clinical manifestation might be multifaceted, including visual and sensory impairment, motor and bladder dysfunction or cognitive problems. The majority of patients ( $85 \%)$ initially present a relapsing remitting (RRMS) disease course characterized by episodes of acute relapses and following remission. Despite great advances within the last decade, above $50 \%$ of untreated RRMS patients go on to develop secondary progressive (SPMS) neurological decline over time.

The molecular mechanisms of MS are thus far not fully elucidated. Infiltration of autoreactive T-cells and release of proinflammatory cytokines lead to inflammatory infiltrates, demyelination, and axonal damage in the CNS as the major pathological findings. ${ }^{1,3}$

As a consequence, most therapeutic approaches targeted the migration of inflammatory cells to the CNS thus dampening the cascade of inflammatory events. However, even if the majority of available therapies may reduce inflammation and relapse frequency, some patients continue to develop progressive neurodegeneration, which in turn drives disability. ${ }^{4}$ Thus, neurodegeneration should be considered as a (c)
hereby accept the Terms. Non-commercial uses of the work are permitted without any further permission from Dove Medical Press Limited, provided the work is properly attributed. For permission for commercial use of this work, please see paragraphs 4.2 and 5 of our Terms (https://www.dovepress.com/terms.php). 
concomitant process that takes place even at disease onset and requires effective medication..$^{5}$ Therefore, there is an unmet need for new therapeutic approaches targeting both inflammation and neurodegeneration, as well as regenerative processes. $^{2}$

Laquinimod is a new orally available agent with numerous mechanisms of action (MoA). ${ }^{6,7}$ In this overview, we review the development of laquinimod, its manifold MoA, and clinical efficacy in published Phase II and III clinical trials. In addition, safety issues, pharmacodynamics, ongoing studies, and future perspectives of laquinimod in MS are discussed.

\section{Development and pharmaco- dynamics of laquinimod}

Laquinimod is a quinoline-3-carboxamide derivate (laboratory code: ABR-215062). It is the result of a structure-activity screening program after commercial launch of its lead compound roquinimex (Linomide ${ }^{\circledR}$ ) was halted owing to unexpected severe cardiac adverse events (AEs) such as serositis, pericarditis, and myocardial infarction observed during Phase III trials. ${ }^{8}$ Chemical modifications of roquinimex led to the discovery of laquinimod. In comparison to roquinimex, laquinimod showed a 20 -fold increase potency in treating animal models of MS and a clearly superior safety profile. ${ }^{9}$

Laquinimod is a once a day oral drug, which is rapidly absorbed in the gut and reaches maximum plasma concentrations approximately within 2 hours of intake. In animal studies, its oral bioavailability was approximately $80 \%-90 \%$. It displays low plasma protein binding and a low rate of total clearance. Laquinimod is metabolized in the liver by the cytochrome isoenzyme CYP3A4 prior to elimination in urine. Only $5 \%-10 \%$ of laquinimod is excreted unchanged. ${ }^{10,11}$ So far, no significant drug interactions are known. However, clinicians should consider interactions with CYP3A4 inducers or inhibitors. ${ }^{12}$ Due to its ability to cross the blood-brain barrier, laquinimod may confer direct protective effects on inflammatory and degenerative CNS processes. ${ }^{11}$

\section{Mode of laquinimod action}

The MoA by which laquinimod exerts its effects are manifold and not yet fully elucidated. Studies conducted in the mouse model of MS, experimental autoimmune encephalomyelitis (EAE), and in vitro studies on peripheral blood mononuclear cells (PBMC) from healthy human donors and patients with MS as well as magnetic resonance imaging (MRI) studies in humans suggest both anti-inflammatory and neuroprotective effects.

\section{Anti-inflammatory effects}

The EAE model is an established animal model of demyelinating CNS inflammation and is widely used to evaluate novel disease-modifying agents in MS research. ${ }^{13}$ In this model, disease severity was mitigated in animals receiving laquinimod either before (preventive treatment) or after disease onset (therapeutic treatment) in a dose-dependent fashion. In comparison to its predecessor substance roquinimex, laquinimod was $\sim 20$ times more effective and its beneficial effect in EAE seems to be independent from endogenous interferon (IFN)-beta. ${ }^{9,11,14}$ Postmortem immunohistochemical analyses demonstrated that laquinimod reduced infiltration of cluster of differentiation (CD) $4^{+}$ $\mathrm{T}$-cells, CD8 ${ }^{+} \mathrm{T}$-cells, and macrophages into the CNS following both treatment regimes. In parallel, there was a reduction of inflammatory demyelination and axonal loss within the CNS compared to control animals. ${ }^{15,16}$

On the level of T-cells, auto aggressive Th17 lymphocytes play a pivotal role in the pathogenesis of MS and EAE. ${ }^{17}$ Laquinimod treatment suppressed the Th17 proinflammatory response in EAE. In addition, Schulze-Topphoff et al ${ }^{18}$ reported on an increase in regulatory $\mathrm{T}$-cells following application of laquinimod. In EAE, there is also evidence that laquinimod further modulates the cytokine balance in favor of an anti-inflammatory environment, characterized by increased levels of interleukin (IL)-4 and IL-10 and decreased levels of IL-12 and tumor necrosis factor (TNF)alpha. Similar results were found in PBMC from healthy volunteers.

Finally, laquinimod may inhibit the entry of leukocytes into the CNS via downregulation of very late antigen (VLA)-4 mediated adhesiveness. ${ }^{16}$ Additionally, laquinimod targets proinflammatory monocytes by reducing inter alia their levels of CD62L and matrix metalloproteinase-9, thus reducing their transmigration into the CNS. 19,20

With regard to antigen presenting cell populations, laquinimod exhibited effects on dendritic cells and monocytes/macrophages. It skewed monocytes toward a regulatory phenotype characterized by increased secretion of IL-10. ${ }^{15,18}$ Laquinimod treatment may also influence dendritic cell maturation and functions in humans and EAE. Treatment of murine dendritic cells with laquinimod caused 1) decreased monocyte chemoattraction and 2) reduced chemokine and cytokine secretion. Jolivel et $\mathrm{al}^{21}$ suggest that inhibition of the nuclear factor $(\mathrm{NF})-\kappa \mathrm{B}$ pathway is responsible for these changes. Effects on the NF- $\kappa B$ pathway have also been reported by others. ${ }^{22}$ In vitro application of laquinimod to B-cells taken from patients with MS 
showed alterations in the expression of genes involved in T-cell activation and NF- $\kappa \mathrm{B}$ pathways. ${ }^{22}$ To investigate potential effects of laquinimod on peripheral blood immune cell populations with a special focus on monocyte phenotype and function, Stasiolek et al analyzed blood samples from 100 patients with RRMS participating in the ALLEGRO trial. Treatment with laquinimod $(0.6 \mathrm{mg} /$ day $)$ resulted in a lower expression of CD86 on monocytes stimulated with lipopolysaccharide. Additionally, upon proinflammatory stimulation monocytes taken from patients in the laquinimod group secreted lower amounts of the proinflammatory chemokines CCL-2 and CCL-5. ${ }^{23}$ Beyond that, laquinimod at a dosage of $0.6 \mathrm{mg}$ orally once daily did not alter the relative proportion of T-cells, B-cells, natural killer (NK)cells, dendritic cells, regulatory T-cells, monocytes, and macrophages after 2 years of continuous treatment. ${ }^{24}$

In an isolated in vitro setting, Kelland et $\mathrm{al}^{25}$ examined the effect of direct exposure of laquinimod on viability, proliferation, migration, and differentiation of neural stem cells and oligodendrocyte progenitor cells of human origin. Direct exposure to laquinimod over a concentration range of $10 \mathrm{nM}$ to $1 \mu \mathrm{M}$ for 24 hours, 48 hours, and 7 days showed no detrimental effects on basic cellular functions of neural stem cells and oligodendrocyte progenitor cells.

\section{Neuroprotective effects}

EAE studies also demonstrated neuroprotective properties of laquinimod. As compared to sham treatment, axonal damage, demyelination, and synaptic loss were reduced in EAE mice treated with laquinimod. ${ }^{10,11}$

Thöne et $\mathrm{al}^{15}$ evaluated the properties of laquinimod to modulate brain-derived neuroprotective factor (BDNF) in 203 patients treated with either laquinimod ( $0.6 \mathrm{mg} /$ day $)$ or placebo. In a blinded analysis, treatment with laquinimod led to a significant and persistent increase in BDNF serum levels compared to placebo treatment. These results were corroborated by EAE experiments. Mice lacking BDNF expression in myeloid cells and T-cells showed a more severe EAE disease course and pronounced axonal damage compared to wildtype animals following laquinimod treatment. Furthermore, Aharoni et $\mathrm{a}^{26}$ reported that treatment of EAE-inflicted mice with laquinimod restored BDNF expression to its level in healthy controls.

Additional evidence supporting the notion that laquinimod may offer neuroprotective effects comes from experiments in cuprizone-induced demyelination in vivo and on primary CNS cells in vitro. Laquinimod targets the CNS-resident inflammatory response of astrocytes via inhibition of $\mathrm{NF}-\kappa \mathrm{B}$, resulting in decreased axonal damage, demyelination, and oligodendrocyte apoptosis. ${ }^{27}$

Laquinimod also displayed neuroprotective activity in EAE on an electrophysiological level by reducing excitatory postsynaptic currents, while increasing inhibitory postsynaptic currents. ${ }^{28}$

\section{Efficacy}

So far, different dosages of laquinimod have been studied in clinical trials in MS and healthy volunteers (Table 1). Here we summarize efficacy and safety findings of published Phase II and III clinical studies investigating laquinimod $(0.1,0.3$, and $0.6 \mathrm{mg} /$ day) in patients with MS. For further information on Phase I studies, we refer to recent reviews. ${ }^{6,7,11}$

\section{Phase II trials}

The first Phase II trial conducted by Polman et al ${ }^{29}$ included 177 patients with RRMS and 33 patients with SPMS and investigated laquinimod at dosages of $0.1 \mathrm{mg} /$ day $(n=68)$ versus $0.3 \mathrm{mg} /$ day $(n=74)$ versus placebo $(n=67)$. The primary endpoint was defined as the mean cumulative number of gadolinium-enhancing (GdE) MRI lesions over 24 weeks. MRI scans were performed every 8 weeks, and 8 weeks after discontinuation of therapy and used a three-time standard dose of gadolinium for the detection of GdE MRI lesions. Secondary analyses included relapse rates and the examination of neurological function using Expanded Disability Status Scale (EDSS) and Multiple Sclerosis Functional Composites (MSFC).

Patients receiving laquinimod $0.3 \mathrm{mg} /$ day displayed a marginally significant $44 \%$ reduction of the mean cumulative number of GdE MRI lesions compared with placebo $(P=0.0498)$. MRI scans done at week 32 observed a trend suggestive of a rebound effect in the laquinimod $0.3 \mathrm{mg} /$ day group. The mean number of clinical exacerbations, the mean EDSS and MSFC did not differ between groups.

A following Phase IIb study was designed to compare the efficacy of two doses of laquinimod ( 0.3 and $0.6 \mathrm{mg} /$ day) versus placebo. The study included a total of 308 patients and was scheduled for 36 weeks. The primary outcome measure was the cumulative number of GdE MRI lesions at weeks $24,28,32$, and 36 . Secondary outcome measures included the total number of confirmed relapses and changes from baseline EDSS during the treatment period. ${ }^{30}$

In contrast to Polman et al, ${ }^{29}$ treatment with laquinimod $0.3 \mathrm{mg} /$ day showed no significant effects, while in subjects receiving $0.6 \mathrm{mg} /$ day laquinimod, the reduction of $\mathrm{GdE}$ MRI lesions reached 40.4\% $(P=0.048)$ compared with 
Table I Clinical efficacy of laquinimod in MS clinical trials

\begin{tabular}{|c|c|c|c|}
\hline Study & Design and drug regimen & Primary endpoint & Results \\
\hline Phase $I^{29}$ & $\begin{array}{l}\text { 24-week, randomized, double-blind, } \\
\text { multicenter } \\
\text { Three arms: LAQ } 0.1 \mathrm{mg} / \text { day, } \\
\text { LAQ } 0.3 \mathrm{mg} / \text { day and placebo } \\
(\mathrm{n}=209)\end{array}$ & $\begin{array}{l}\text { Mean cumulative number of GdE } \\
\text { MRI lesions over } 24 \text { weeks }\end{array}$ & $\begin{array}{l}\text { Significant reduction by } 44 \% \text { in the group } \\
\text { treated with LAQ }(0.3 \mathrm{mg} / \text { day }) \text { when } \\
\text { compared to placebo } \\
\text { ARR: no significant difference between groups }\end{array}$ \\
\hline $\begin{array}{l}\text { Phase } \mathrm{Ilb}^{30} \\
(\mathrm{LAQ} / 5062)\end{array}$ & $\begin{array}{l}\text { 36-week, randomized, double-blind, } \\
\text { multicenter } \\
\text { Three arms: LAQ } 0.3 \mathrm{mg} / \text { day, } \\
\text { LAQ } 0.6 \mathrm{mg} / \text { day, and placebo } \\
(\mathrm{n}=308)\end{array}$ & $\begin{array}{l}\text { Cumulative number of GdE } \\
\text { MRI lesions at weeks } 24,28,32 \text {, } \\
\text { and } 36\end{array}$ & $\begin{array}{l}\text { Significant reduction by } 40.4 \% \text { and } 44 \% \text { in } \\
\text { cumulative number of GdE MRI lesions and } \\
\text { new T2 lesions, respectively, for LAQ } \\
(0.6 \mathrm{mg} / \text { day) as compared to placebo. LAQ } \\
0.3 \mathrm{mg} / \text { day showed no significant effects } \\
\text { ARR: no significant difference between groups }\end{array}$ \\
\hline $\begin{array}{l}\text { Phase } \mathrm{Ilb}^{31} \\
(\mathrm{LAQ} / 5063)\end{array}$ & $\begin{array}{l}\text { 36-week, multicenter, double-blind } \\
\text { extension of LAQ/5062. Subjects on } \\
\text { placebo were switched to LAQ } 0.3 \text { or } \\
0.6 \mathrm{mg} / \text { day, while the treatment arm } \\
\text { subjects were continued on LAQ } \\
(\mathrm{n}=257)\end{array}$ & $\begin{array}{l}\text { Number of GdE MRI lesions, } \\
\text { new T2 lesions, volume of T2 } \\
\text { lesions, and new hypointense } \\
\text { TI lesions over the length of } \\
\text { the study. MRI scans were } \\
\text { performed at baseline and at } \\
\text { week } 36\end{array}$ & $\begin{array}{l}\text { Subjects switched to LAQ showed a } \\
\text { significant reduction by } 52 \% \text { in the mean } \\
\text { number of GdE MRI lesions between the } \\
\text { start and the end of the extension phase. } \\
\text { Treatment effect was more significant in } \\
\text { subjects switched to LAQ } 0.6 \mathrm{mg} / \text { day. } \\
\text { Subjects that continued on the treatment } \\
\text { group showed sustained benefit throughout } \\
\text { the extension. } \\
\text { ARR: no significant difference between groups }\end{array}$ \\
\hline $\begin{array}{l}\text { ALLEGRO32 } \\
\text { (Phase III) }\end{array}$ & $\begin{array}{l}\text { 24-month, randomized, double-blind, } \\
\text { multicenter } \\
\text { Two arms: LAQ } 0.6 \mathrm{mg} / \text { day and placebo } \\
\text { ( } \mathrm{n}=\mathrm{I}, 106 \text { ) } \\
\text { (LAQ: } 550 \text { vs placebo: } 556 \text { ) }\end{array}$ & ARR at 24 months & $\begin{array}{l}\text { Significant but modest reduction in ARR for } \\
\text { the treatment group compared to placebo } \\
(0.30 \text { vs } 0.39 \text {, respectively; } P=0.002)\end{array}$ \\
\hline $\begin{array}{l}\text { BRAVO }^{33} \\
\text { (Phase III) }\end{array}$ & $\begin{array}{l}\text { 24-month, randomized, double-blind, } \\
\text { multicenter } \\
\text { Three arms: LAQ } 0.6 \mathrm{mg} / \text { day, IFN- } \beta \text { Ia } \\
\text { im and placebo ( } \mathrm{n}=1,33 \mathrm{I}) \\
\text { (LAQ: } 434 \text { vs placebo: } 450 \text { vs IFN- } \beta \text { : } 447 \text { ) }\end{array}$ & ARR at 24 months & $\begin{array}{l}\text { No significant effect. Treatment with LAQ } \\
0.6 \mathrm{mg} / \text { day } \\
\text { showed a trend to reduction in ARR } \\
(P=0.075) \text {. Following an adjustment for } \\
\text { imbalance of baseline MRI disease activity } \\
\text { between groups, LAQ }(0.6 \mathrm{mg} / \text { day }) \\
\text { significantly reduced ARR when compared to } \\
\text { placebo }\end{array}$ \\
\hline
\end{tabular}

Abbreviations: MS, multiple sclerosis; ARR, annualized relapse rate; GdE, gadolinium enhancing; IFN, interferon; MRI, magnetic resonance imaging; LAQ, laquinimod.

placebo. ${ }^{29,30}$ Over the short study period, both dosages did not demonstrate significant effects on relapse rates and EDSS. Two hundred and fifty-seven patients of the core LAQ/5062 trial were enrolled in a 36-week double-blind extension study (LAQ/5063). Subjects originally on placebo were switched to laquinimod 0.3 or $0.6 \mathrm{mg} /$ day, while individuals in the treatment arm were continued on laquinimod. Subjects switched to laquinimod showed a significant reduction by $52 \%$ in the mean number of GdE MRI lesions between baseline and week 36. Subjects who continued on the treatment group showed sustained benefit throughout LAQ/5063. In neither group, EDSS or MSFC changed significantly. ${ }^{31}$

\section{Phase III trials}

So far, the efficacy of $0.6 \mathrm{mg} /$ day laquinimod in patients with RRMS was analyzed in two Phase III studies, ALLEGRO (Assessment of Oral Laquinimod in Preventing
Progression in Multiple Sclerosis; \#NCT00509145) and BRAVO (Benefit-Risk Assessment of Avonex and Laquinimod; \#NCT00605215). ${ }^{32,33}$ Both multicenter studies were scheduled for 24 months, placebo-controlled, randomized, and double-blind. In BRAVO a third group of patients received IFN-beta 1a once a week intramuscular (Avonex ${ }^{\circledR}$ ). However, it is important to note that BRAVO was not designed to provide a direct statistical comparison between laquinimod and IFN-beta 1a. The primary outcome measure in ALLEGRO and BRAVO was the annualized relapse rate (ARR) during the 24-month follow-up period.

In comparison to placebo, ALLEGRO demonstrated a modest but significant reduction of the mean ARR in subjects treated with $0.6 \mathrm{mg}$ /day laquinimod ( 0.30 vs 0.39 , $P=0.002) .{ }^{32}$ In parallel, a higher proportion of patients in the treatment group was relapse-free and showed a reduced risk of confirmed disability progression (defined as a 3-month 
sustained increase in EDSS score) compared with placebo (62.9\% vs $52.2 \% ; P<0.001$ and $11.1 \%$ vs $15.7 \% ; P=0.01$, respectively). Similar to Phase II studies, laquinimod showed significant effects on secondary MRI endpoints including the total number of GdE MRI lesions (1.33 vs 2.12; $P<0.001)$ and new or enlarging lesions on T2-weighted MRI (5.03 vs $7.14 ; P<0.001)$. Apart from that, laquinimod slowed brain volume loss from baseline during the study period as compared with placebo.

Additional evidence supporting the neuroprotective capacity of laquinimod comes from brain MRI studies in ALLEGRO. Here, laquinimod led to lower rates of whitematter and thalamic atrophy at months 12 and 24, gray-matter atrophy at month 12 , and a trend for less gray-matter atrophy at month 24. Other MRI techniques sensitive to neuronal damage, including magnetization transfer ratio imaging of white-matter, gray-matter, and normal appearing tissue, may further support laquinimod's neuroprotective MoA. ${ }^{34}$

In BRAVO, laquinimod missed the primary outcome measure of reducing ARR compared to placebo. ${ }^{33}$ However, following adjustment for an imbalance between groups in the volume of baseline T2 disease and the number of GdE MRI lesions, laquinimod reduced the ARR by $21 \%$ ( 0.29 vs 0.37 , $\mathrm{RR}=0.787$, 95\% confidence interval $[\mathrm{CI}]=0.637-0.972$; $P=0.026)$. Secondary analysis of the BRAVO study included percent brain volume change and confirmed disability progression as measured by the EDSS. The percentage of brain volume loss was less pronounced in the laquinimod group $(21 \% ; P<0.001)$, while effects on confirmed disability progression became statistically significant after adjustment for imbalance $(P=0.044)$.

\section{Safety issues}

The first Phase II study by Polman et $\mathrm{al}^{29}$ showed a favorable safety profile and reported on a similar proportion of patients with AEs in the three treatment arms. The favorable safety profile was confirmed in LAQ/5062 and its extension study. ${ }^{30,31}$ Both studies reported no deaths, and the incidence of AE did not differ between groups. AEs (except elevated liver enzymes) more frequently seen in LAQ/5062 in subjects receiving laquinimod were chest pain, arthralgia, and herpes simplex as well as herpes zoster infections. The overall number of infections was similar between groups. In $\mathrm{LAQ} / 5062$, the incidence of serious $\mathrm{AE}$ was $4.9 \%$ for placebo, $2.8 \%$ for laquinimod $0.6 \mathrm{mg} /$ day, and $5.1 \%$ for laquinimod $0.3 \mathrm{mg} /$ day. During the 36 -week Phase II active extension study, no life threatening/opportunistic infections or malignancies occurred. Patients switched to laquinimod showed a similar incidence of AE as compared to subjects originally treated with laquinimod. ${ }^{31}$

In ALLEGRO, $87 \%$ in the laquinimod and $81 \%$ in the placebo group reported at least one AE. Most common AE seen at a higher incidence (except elevated liver enzymes) were back pain, cough, arthralgia and abdominal pain (Table 2). ${ }^{32}$ With regard to serious $\mathrm{AE}, 11.1 \%$ in the laquinimod group had a serious $\mathrm{AE}$ as compared to $9.5 \%$ of the placebo group. No death, no case of fatal liver failure, and no increased likelihood of herpes virus infections or neoplasms (eight in the laquinimod group vs six in the placebo group) were reported in ALLEGRO. ${ }^{32}$ Serious AEs seen more frequently in the laquinimod group than in subjects receiving placebo are shown in Table 2. Remarkably, there was a higher incidence of appendicitis in subjects treated with laquinimod.

Safety data from the BRAVO study demonstrated a similar safety profile to that of ALLEGRO ${ }^{33}$ Similar proportions of subjects treated with either laquinimod, placebo, or IFN-beta 1a were discontinued early due to AE (laquinimod $5 \%$ vs placebo $4 \%$ vs IFN-beta 1 a $6 \%$; BRAVO). Most common AEs (except elevated liver enzymes) seen in subjects treated with laquinimod versus placebo were abdominal pain, headache, and nausea. The number of severe AEs did not differ significantly between groups. Additionally, two deaths occurred, one in the laquinimod and one in the IFNbeta 1a group, however, both were assessed by the investigator as unrelated to the study medication. There was one case of superficial thrombophlebitis in a subject treated with laquinimod (BRAVO). ${ }^{33}$

In terms of laboratory values, laquinimod led to an elevation of liver enzymes (alanine transaminase and aspartate transaminase) in comparison to placebo treatment in Phase II

Table 2 Overview of safety issues

\begin{tabular}{lll}
\hline Adverse events & Changes in laboratory values & Safety precautions \\
\hline Elevation of liver enzymes & Elevation of liver enzymes & $\begin{array}{l}\text { Monitoring for liver enzymes } \\
\text { and signs of infection }\end{array}$ \\
$\begin{array}{l}\text { Abdominal and back pain, cough, } \\
\text { dizziness, headache, diarrhea, }\end{array}$ & $\begin{array}{l}\text { Shift of C-reactive protein (CRP) } \\
\text { and respiratory tract infections }\end{array}$ & \\
\hline
\end{tabular}


and III studies. ${ }^{29-33}$ Typically, elevated liver enzymes occurred within the first 6 months of treatment and were reversible over the course of the study without withdrawing therapy. If elevated liver enzymes led to discontinuation of the study drug in any way, they normalized within 2 months (ALLEGRO). ${ }^{32}$ There was no concomitant increase in bilirubin levels or change in coagulation tests (ALLEGRO; BRAVO). ${ }^{32,33}$ In the LAQ/5062 trial, shifts in C-reactive protein and fibrinogen were monitored and showed no significant change. ${ }^{30}$

To date, there are no reliable data concerning teratogenic effects in humans. Nevertheless, in women of child bearing age, a strict use of contraceptives is advisable, and breastfeeding women should not be exposed to laquinimod. An increased risk of fetal malformations has been reported in rats following treatment with other quinoline derivatives. ${ }^{35}$

In summary, based on Phase II and III trials available to date, laquinimod $(0.1,0.3$, and $0.6 \mathrm{mg} /$ day $)$ has been well tolerated in patients with MS. The most common AE following treatment with laquinimod was a dose-dependent elevation of liver enzymes. In comparison to its lead compound roquinimex, laquinimod showed a clearly superior safety profile, with no apparent evidence to induce cardiac AEs.

\section{Regulatory affairs}

Laquinimod received a negative opinion from the Committee for Medicinal Products for Human Use (CHMP) of the European Medicines Agency (EMA) in 2014. ${ }^{36}$ In particular, the CHMP expressed concerns regarding a higher occurrence of cancers after long-term exposure to the compound in animal studies and noted that a similar long-term cancer risk could not be sufficiently excluded in humans, although there were no signals of malignancy in the clinical trials. The Committee also discussed possible pregnancy risks, again based on data from animal studies, yet without any signals from human trials where strict contraception was advised. Based on the data available, the CHMP concluded that the benefits of laquinimod at $0.6 \mathrm{mg} /$ day were not sufficient to outweigh the potential risks in patients with RRMS and refused marketing authorization at that time.

In response to a request from the US Food and Drug Administration, Teva Pharmaceuticals rolled out a third Phase III trial (CONCERTO) in subjects with RRMS. CONCERTO is designed to evaluate the efficacy, safety, and tolerability of two doses of laquinimod 0.6 or $1.2 \mathrm{mg} /$ day in subjects with RRMS (see later).

\section{Ongoing trials}

CONCERTO (The Efficacy and Safety and Tolerability of Laquinimod in Subjects With Relapsing Remitting Multiple
Sclerosis [\#NCT01707992]) is a third randomized, doubleblind, parallel-group, placebo-controlled Phase III study designed to evaluate the efficacy, safety, and tolerability of two doses of laquinimod 0.6 or $1.2 \mathrm{mg} /$ day in RRMS. The study is scheduled for 24 months, currently ongoing but not recruiting participants. The primary outcome measure is the time to confirmed disease progression as measured by EDSS. Secondary outcome measures are percent change in brain volume from baseline to month 15 and time to first confirmed relapse. The estimated study completion date is April 2019. Additionally, three extension studies (\#NCT00745615, \#NCT00988052, and \#NCT01047319) are currently ongoing in order to evaluate the long-term safety, tolerability, and effect of $0.6 \mathrm{mg} /$ day laquinimod in RRMS.

Due to laquinimod's suggested effect on neurodegenerative aspects a Phase II study in subjects with primary progressive MS (ARPEGGIO, \#NCT02284568) evaluating two doses of laquinimod 0.6 and $1.5 \mathrm{mg}$ /day as compared to placebo is now underway.

The compound is also tested in neurodegenerative disease: The LEGATO trial is a multicenter, multinational, randomized, double-blind, placebo controlled, parallel group study to assess the efficacy and safety of laquinimod at dosages of $0.5,1.0$, and $1.5 \mathrm{mg} /$ day for the treatment of patients with clinically proven Huntington's disease (HD) and/or positive genetic testing with 36-49 CAG repeats in the Huntingtin gene. The primary endpoint is a change from baseline in the motor score of the Unified HD Rating Scale over 12 months. The study will enroll 400 patients, and study completion is expected in January 2017.

\section{Expert opinion}

Laquinimod is an orally available new compound under development for MS and neurodegenerative disease. Approximately 10 years ago, there was much enthusiasm about the introduction of new oral compounds to MS therapy with five oral medications under development in Phase III trials (cladribine, dimethyl fumarate, fingolimod, laquinimod, and teriflunomide). Yet, finally, only two of these compounds (dimethyl fumarate and teriflunomide) received marketing authorization in the European Community as first-line therapies for RRMS. Still, even these compounds face challenges like the occurrence of progressive multifocal eukoencephalopathy under treatment with dimethyl fumarate or the need for strict contraception and the very long half-life requiring wash out procedures in the case of teriflunomide. Thus, there is a persisting, but still insufficiently met, need for safe and at the same time effective oral compounds in MS therapy, especially regarding chronic-progressive disease forms and 
neuroprotective effects. Here, laquinimod may offer an interesting MoA with pleiotropic effects on several cell types also comprising actions on CNS cells such as astrocytes.

In Phase III clinical trials in RRMS, effects of laquinimod on relapse rates were modest, and in some cases, only significant after adjusting the data. Yet, the laquinimod dosage of $0.6 \mathrm{mg}$ once daily might have been too low to achieve optimal immunomodulatory effects. It is reassuring that the company started testing with low dosages given the cardiac side effects of the predecessor roquinimex. However, dose titration studies revealed a good tolerability of laquinimod also at higher dosages where an improved immunomodulatory efficacy may be observed. This concept is now tested in the CONCERTO trial. Even more interesting are the consistent effects of laquinimod on disease progression and brain atrophy, which seem to be somehow uncoupled from effects on relapse rates. It is tempting to speculate that these effects may be due to a direct neuroprotective action of the compound as suggested by animal studies. Consequently, this led to clinical trials of laquinimod in primary progressive MS (ARPEGGIO trial) and also HD (LEGATO trial) as conditions where neurodegenerative mechanism may prevail over systemic inflammation. This approach seems particularly worthwhile given the even higher unmet need for causal therapies in these devastating conditions with no causal treatment or even cure available so far. This is of particular interest in HD, which may serve as a well-chosen prototype for primary neurodegeneration with astroglial pathology and - given the available genetic testing - a defined patient population with a well-characterized presymptomatic disease phase.

All these trials will also help to shed further light on the safety profile of laquinimod. A complete set of safety data has become an important and indispensable part of a new compound's profile prior to marketing authorization. This holds particularly true in RRMS, where several immunomodulators are already available in the market, some with a long-standing and well-characterized safety profile like beta IFNs and glatiramer acetate. Yet, in clinical practice of RRMS treatment, long-term safety with new oral compounds does remains a concern in a predominantly young patient population, particularly regarding opportunistic infections and malignancy. While preliminary data in rodents may be helpful to gain first insights into the toxicity of new medications, they are also limited with regard to metabolization and particularly long-term effects. Thus, comprehensive safety data sets, including further data from human trials with laquinimod, may be the best way to refute concerns expressed by the regulatory authorities.
Conclusion

Laquinimod offers the perspective to add to the therapeutic armamentarium in patients with MS and neurodegeneration. Current data show that at the dose tested, laquinimod demonstrated a modest effect on relapse rates but no superiority to established disease-modifying treatments in RRMS. Yet, effects on disease progression and brain atrophy, which has been recognized as a surrogate marker of neuronal damage in MS, were more convincing.

In all trials, laquinimod was generally well tolerated. Importantly, no signs of severe cardiac AE were observed. The proposed MoA whereby laquinimod ameliorates disease progression in MS is manifold and may include neuroprotective actions.

Currently, further studies are ongoing to evaluate the safety and efficacy of laquinimod at a higher dose (CONCERTO) as well as in subjects with primary progressive MS (ARPEGGIO) or neurodegenerative disease such as HD (LEGATO). Results from these trials will further inform about the clinical benefit of laquinimod in patient populations with a persisting, but still insufficiently met, need for safe and at the same time effective oral compounds with neuroprotective effects.

\section{Acknowledgments}

JT conducted research projects sponsored by Teva Pharmaceuticals. RAL received travel expenses and speaker and research Honoria from Biogen, Genzyme, MerckSerono, Novartis Pharma, Roche, and TEVA Pharma. RAL holds an endowed professorship supported from the Novartis Foundation for Therapeutic Research.

\section{Disclosure}

The authors report no conflicts of interest in this work.

\section{References}

1. Nylander A, Hafler DA. Multiple sclerosis. J Clin Invest. 2012;122(4): $1180-1188$.

2. Hauser SL, Chan JR, Oksenberg JR. Multiple sclerosis: prospects and promise. Ann Neurol. 2013;74(3):317-327.

3. Grigoriadis N, van Pesch V, ParadigMS Group. A basic overview of multiple sclerosis immunopathology. Eur J Neurol. 2015;22 Suppl 2:3-13.

4. De Stefano N, Airas L, Grigoriadis N, et al. Clinical relevance of brain volume measures in multiple sclerosis. CNS Drugs. 2014;28(2): 147-156.

5. Kutzelnigg A, Lassmann H. Pathology of multiple sclerosis and related inflammatory demyelinating diseases. Handb Clin Neurol. 2014;122: 15-58.

6. Kolb-Sobieraj C, Gupta S, Weinstock-Guttman B. Laquinimod therapy in multiple sclerosis: a comprehensive review. Neurol Ther. 2014;3(1): 29-39.

7. Thöne J, Gold R. Review of laquinimod and its therapeutic potential in multiple sclerosis. Expert Opin Pharmacother. 2013;14(18): 2545-2552. 
8. Noseworthy JH, Wolinsky JS, Lublin FD, et al. Linomide in relapsing and secondary progressive MS: part I: trial design and clinical results. North American Linomide Investigators. Neurology. 2000;54(9): 1726-1733.

9. Brunmark C, Runstrom A, Ohlsson L, et al. The new orally active immuno regulator laquinimod (Abr-215062) effectively inhibits development and relapses of experimental autoimmune encephalomyelitis. J Neuroimmunol. 2002;130:163-172.

10. Thöne J, Gold R. Laquinimod: a promising oral medication for the treatment of relapsing-remitting multiple sclerosis. Expert Opin Drug Metab Toxicol. 2011;7(3):365-370.

11. Brück W, Wegner C. Insight into the mechanism of laquinimod action. J Neurol Sci. 2011;306(1-2):173-179.

12. Tuvesson H, Hallin I, Persson R, Sparre B, Gunnarsson PO, Seidegård J. Cytochrome P450 3A4 is the major enzyme responsible for the metabolism of laquinimod, a novel immunomodulator. Drug Metab Dispos. 2005;33(6):866-872.

13. Linker RA, Lee DH. Models of autoimmune demyelination in the central nervous system: on the way to translational medicine. Exp Transl Stroke Med. 2009;1:5.

14. Runström A, Leanderson T, Ohlsson L, Axelsson B. Inhibition of the development of chronic experimental autoimmune encephalomyelitis by laquinimod (ABR-215062) in IFN-beta k.o. and wild type mice. J Neuroimmunol. 2006;173(1-2):69-78.

15. Thöne J, Ellrichmann G, Seubert S, et al. Modulation of autoimmune demyelination by laquinimod via induction of brain-derived neurotrophic factor. Am J Pathol. 2012;180(1):267-274.

16. Wegner C, Stadelmann C, Pförtner R, et al. Laquinimod interferes with migratory capacity of $\mathrm{T}$ cells and reduces IL-17 levels, inflammatory demyelination and acute axonal damage in mice with experimental autoimmune encephalomyelitis. J Neuroimmunol. 2010; 227(1-2):133-143.

17. Rostami A, Ciric B. Role of Th17 cells in the pathogenesis of CNS inflammatory demyelination. J Neurol Sci. 2013;333(1-2):76-87.

18. Schulze-Topphoff U, Shetty A, Varrin-Doyer M, et al. Laquinimod, a quinoline-3-carboxamide, induces type II myeloid cells that modulate central nervous system autoimmunity. PLoS One. 2012;7(3):e33797.

19. Mishra MK, Wang J, Silva C, Mack M, Yong VW. Kinetics of proinflammatory monocytes in a model of multiple sclerosis and its perturbation by laquinimod. Am J Pathol. 2012;181(2):642-651.

20. Mishra MK, Wang J, Keough MB, et al. Laquinimod reduces neuroaxonal injury through inhibiting microglial activation. Ann Clin Transl Neurol. 2014;1(6):409-422.

21. Jolivel V, Luessi F, Masri J, et al. Modulation of dendritic cell properties by laquinimod as a mechanism for modulating multiple sclerosis. Brain. 2013;136(Pt 4):1048-1066.

22. Gurevich M, Gritzman T, Orbach R, Tuller T, Feldman A, Achiron A. Laquinimod suppresses antigen presentation in relapsing-remitting multiple sclerosis: in-vitro high-throughput gene expression study. J Neuroimmunol. 2010;221(1-2):87-94.
23. Stasiolek M, Linker RA, Hayardeny L, Bar Ilan O, Gold R. Immune parameters of patients treated with laquinimod, a novel oral therapy for the treatment of multiple sclerosis: results from a double-blind placebo-controlled study. Immun Inflamm Dis. 2015;3(2):45-55.

24. Lund BT, Kelland EE, Hayardeny L, Barilan O, Gilmore W, Weiner LP. Assessment of changes in immune measures of multiple sclerosis patients treated with laquinimod. J Neuroimmunol. 2013;263(1-2):108-115.

25. Kelland EE, Gilmore W, Hayardeny L, Weiner LP, Lund BT. In vitro assessment of the direct effect of laquinimod on basic functions of human neural stem cells and oligodendrocyte progenitor cells. J Neurol Sci. 2014;346(1-2):66-74.

26. Aharoni R, Saada R, Eilam R, Hayardeny L, Sela M, Arnon R. Oral treatment with laquinimod augments regulatory $\mathrm{T}$-cells and brain-derived neurotrophic factor expression and reduces injury in the CNS of mice with experimental autoimmune encephalomyelitis. J Neuroimmunol. 2012;251(1-2):14-24.

27. Brück W, Pförtner R, Pham T, et al. Reduced astrocytic NF- $\kappa$ B activation by laquinimod protects from cuprizone-induced demyelination. Acta Neuropathol. 2012;124(3):411-4-24.

28. Ruffini F, Rossi S, Bergamaschi A, et al. Laquinimod prevents inflammation-induced synaptic alterations occurring in experimental autoimmune encephalomyelitis. Mult Scler. 2013;19(8):1084-1094.

29. Polman C, Barkhof F, Sandberg-Wollheim M, et al. Treatment with laquinimod reduces development of active MRI lesions in relapsing MS. Neurology. 2005;64(6):987-991.

30. Comi G, Pulizzi A, Rovaris M, et al. Effect of laquinimod on MRImonitored disease activity in patients with relapsing-remitting multiple sclerosis: a multicentre, randomised, double-blind, placebo-controlled phase IIb study. Lancet. 2008;371(9630):2085-2092.

31. Comi G, Abramsky O, Arbizu T, et al. Oral laquinimod in patients with relapsing-remitting multiple sclerosis: 36 -week double-blind active extension of the multi-centre, randomized, double-blind, parallel-group placebo-controlled study. Mult Scler. 2010;16(11):1360-1366.

32. Comi G, Jeffery D, Kappos L, et al; ALLEGRO Study Group. Placebo-controlled trial of oral laquinimod for multiple sclerosis. $\mathrm{NEngl}$ J Med. 2012;366(11):1000-1009.

33. Vollmer TL, Sorensen PS, Selmaj K, et al; BRAVO Study Group. A randomized placebo-controlled phase III trial of oral laquinimod for multiple sclerosis. J Neurol. 2014;261(4):773-783.

34. Filippi M, Rocca MA, Pagani E, et al. Placebo-controlled trial of oral laquinimod in multiple sclerosis: MRI evidence of an effect on brain tissue damage. J Neurol Neurosurg Psychiatry. 2014;85(8):851-858.

35. Bjork A, Jonsson A, Fex T, Hedlund G. inventors; Active Biotech Ab. assignee. Quinoline derivatives. United States Patent US 6593343 B2. 2003 July 15.

36. European Medicine Agency. Refusal of the marketing authorisation for Nerventra (laquinimod). European Medicine Agency; 2014. Available from: http://www.ema.europa.eu/docs/en_GB/document_library/ Summary_of_opinion_-_Initial_authorisation/human/002546/ WC500160120.pdf. Accessed February 18, 2016.
Drug Design, Development and Therapy

\section{Publish your work in this journal}

Drug Design, Development and Therapy is an international, peerreviewed open-access journal that spans the spectrum of drug design and development through to clinical applications. Clinical outcomes, patient safety, and programs for the development and effective, safe, and sustained use of medicines are a feature of the journal, which

\section{Dovepress}

has also been accepted for indexing on PubMed Central. The manuscript management system is completely online and includes a very quick and fair peer-review system, which is all easy to use. Visit http://www.dovepress.com/testimonials.php to read real quotes from published authors. 